\title{
RESUMEN
}

Este artículo pretende mostrar una serie de variables e indicadores que proporcionan las empresas de tipo micro, pequeñas y medianas empresas (PYMES) en cantones y distritos de la Región del Pacífico Central. Se evalúan elementos como: caracterización del dueño de la empresa, caracterización de las Pymes, actividades desarrolladas, valor de los ingresos netos, áreas que se desean optimizar, necesidades de acompañamientos, entre otros elementos. La finalidad, de este estudio, consiste en identificar las debilidades y fortalezas que permitan desarrollar planes de acción, para incrementar su participación en el mercado costarricense, mediante el apoyo del TCU653, de la Universidad de Costa Rica, Sede del Pacífico.

Palabras clave: Pymes- diagnóstico- retos- soluciones- TCU

\section{DIAGNÓSTICOS DE MICRO, PEQUEÑA Y MEDIANA EMPRESA EN LA REGIÓN PACÍFICO CENTRAL- COSTA RICA, 2019.}

\author{
Kathia Cousin Brenes ${ }_{1} /$ Rafael Alvarado Gamboa
}

\section{Fechas de recepción: 30 de octubre de 2019 \\ Fecha de aceptación: 8 de diciembre de 2019}

\section{DOI: https://doi.org/10.22370/riace.2019.8.1.2074}

1 Licenciatura en Contaduría Pública con énfasis en Administración de Negocios, Maestría en Administración de Negocios con énfasis en Gerencia Estratégica. Doctorada en Estudios de la Sociedad y la Cultura de la Universidad de Costa Rica. Profesora en Sede del Pacífico de la Universidad de Costa Rica, País Costa Rica/ América Central. Desde 1998.

2 Licenciatura en Contaduría Pública de la Universidad Estatal a Distancia. Profesor de la Sede del Pacífico de la Universidad de Costa Rica, País Costa Rica /América Central. Desde el 2013. 


\section{DIAGNOSTICS OF MICRO, SMALL AND MEDIUM-SIZED ENTERPRISES IN THE CENTRAL PACIFIC REGION- COSTA RICA, 2019.}

\section{ABSTRACT}

This article aims to show a sequence of variables and indicators provided by small and medium-sized enterprises (SMEs), located in the central pacific region. Some of the variables analyzed are: the gender of the owner of the business, characterization of the SMEs, type of developed activities, annual turnover, areas they want to improve, required trainings, between others. The purpose, of this study, is to identify the strengths and weaknesses, that will enable to develop action plans to increase the empowerment of this business in Costa Rican market, through University of Costa Rica's TCU 653.

KEYWORDS: Pymes- SMEs- diagnostic- challenges- solutions- TCU 


\section{INTRODUCCIÓN}

Costa Rica, un país ubicado en Centroamérica, aplica el modelo ${ }_{3}$ de desarrollo denominado promoción de exportaciones o liberación económica, enfocado en promocionar las exportaciones de productos no tradicionales y atraer la inversión extranjera. De igual manera, el país promueve, principalmente, a través de las acciones de oferta y demanda de los mercados ${ }_{4}$ una economía capitalista. Sin embargo, el debate entre las ventajas y las desventajas del modelo es complejo.

Hoy, Costa Rica se ve obligado a dar respuesta a los desafíos locales y globales (Cousin, 2018). En esa línea, precisamente su sector económico está constituido por un tejido empresarial heterogéneo, compuesto por empresas micro, pequeñas y medianas denominadas Pymes $_{5}$. Estas empresas generan empleo, aumentan la calidad de vida de los costarricenses y permiten un manejo sostenible de la economía (Chen y García, 2012; Carvajal, 2014; Cousin, 2015). Así, en ese orden de ideas, el aumento del número de Pymes implica un aumento en actividades comerciales, industriales, de turismo o servicios en diferentes sectores de la economía que beneficia a toda la sociedad.

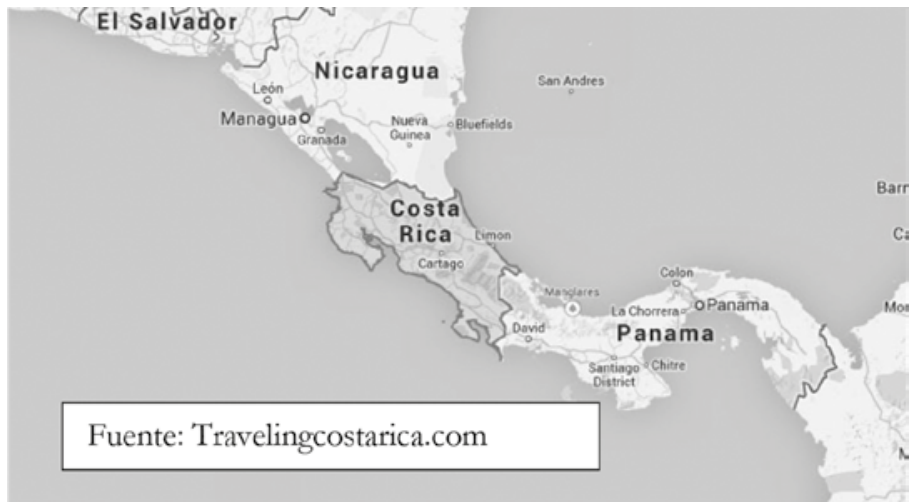

Durante los últimos años, las Pymes registradas ${ }_{6}$ en Costa Rica se han multiplicado, de acuerdo con el Ministerio de Economía Industria y Comercio (MEIC, 2017), pues

3 Para aclarar al lector, los modelos desarrollo son llamados también enfoques de políticas (Cousin, 2018).

4 El mercado es un proceso en el cual las sociedades realizan intercambios entre bienes y servicios los cuales satisfacen necesidades.

5 Por conveniencia de la investigación, se comprenderá como micro, aquella empresa que emplea de 1 a 5 personas, a la pequeña a la que emplea de 6 a 30 personas, por mediana aquella empresa que emplea de 31 a 100 empleados (CCSS, 2018 y Observatorio, 2010). Además, se incluye que, para efectos del trabajo, el término mipyme está contenido en la definición pyme, véase decreto ejecutivo ํ3311, del 06 de abril de 2006.

Se aclara que el Ministerio de Economía, posee otra clasificación en base a las ventas, sin embargo, por la vulnerabilidad de las ventas en esta región decidimos no tomar esta clasificación.

6 En cuanto a este tema, no es posible tener un dato exacto sobre la cantidad de pymes informales que existen en Costa Rica y tampoco en la Región Pacífico Central. 
representan un 93,3\% del total de empresas a nivel nacional. Contrario a esto, las Pymes van descendiendo o disminuyendo, desde 2006, en cuanto a su tasa de crecimiento (Cousin, 2015). ¿Qué podemos hacer? Es claro, para la supervivencia y el sostenimiento de las Pymes, se deben implementar habilidades y estrategias para identificar una nueva manera de reinventarse o innovar constantemente en un entorno vasto y complejo.

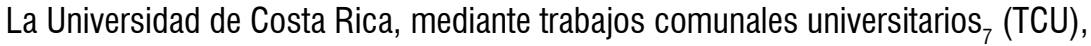
en especial en la Sede del Pacífico de la provincia de Puntarenas, se ha preocupado por fortalecer los procesos empresariales con el propósito de mejorar la economía local. El interés por realizar un análisis exploratorio de este sector en la Región Pacífico Central, se justifica para posteriormente ofrecer posibilidades objetivas a sus necesidades. Agregando, que existen pocos estudios sobre el tema en la región (Chen y García, 2012).

Para tal efecto, esta investigación analiza las siguientes categorías empresariales: Identificación de la empresa, Estructura, Actividades desarrolladas, Administración de la información, Información del Mercadeo y Mejora Continua.

\section{MATERIALES Y MÉTODOS}

Como un antecedente, la Región Pacífico Central en Costa Rica presenta un índice de pobreza del 29,9\%, según el informe presentado por el Estado de la Nación y el Instituto Nacional de Estadística y Censo (INEC, 2018), lo cual dificulta las posibilidades de desarrollo en la zona. Relacionado a lo anterior, esto genera un estancamiento en la economía local y se refleja en los problemas económicos y sociales que se presentan.

El proyecto Trabajo Comunal Universitario (TCU) 653: Acompañamiento y fortalecimiento de las áreas administrativas y técnicas al parque empresarial Pymes de la Región Pacífico Central, se ha preocupado por realizar asesorías empresariales, para intentar que las Pymes locales logren crecer en un mundo competitivo. Sin embargo, debe enfatizarse que, era necesario construir una base de datos propia de la Universidad de Costa Rica, para la región, por las pocas opciones de información en la zona.

En ese orden, la investigación tiene como principal objetivo realizar un análisis exploratorio de las características principales de las Pymes de la Región del Pacífico Central, con el objetivo de a diseñar una estrategia por medio del TCU 653, para ayudar y fortalecer este sector de la economía costarricense.

7 En Costa Rica, la Universidad de Costa Rica posee los TCU o trabajos comunales universitarios. Lo cual es una actividad que vincula grupos y comunidades vulnerables con la población estudiantil con el objetivo de desarrollar un proceso académico multidisciplinario e interdisciplinario, que integre actividades sustantivas de la Universidad de manera solidaria con sus necesidades. El cual consta de 300 horas de trabajo, sin excepción para que cada estudiante pueda graduarse de un plan de bachillerato universitario. La coordinación de este proyecto de TCU estaría a cargo de un profesor universitario (Reglamento de Trabajo Comunal Universitario de la Universidad de Costa Rica, 2018). 
Vale la pena aclarar que los métodos de investigación que se emplearon en dicho proyecto fueron de carácter tanto cualitativo como cuantitativo. En relación al método cuantitativo, su importancia radica que, al momento de la elaboración de mediciones estadísticas basados en una población específica, permiten cuantificar o medir los fenómenos y sus efectos para sacar posibles conclusiones (Sampieri, Méndez, Mendoza y Cuevas, 2017). En cambio, para estos autores, el método cualitativo puede emplearse para explorar 0 describir percepciones y experiencias desde la perspectiva de los participantes de las Pymes a interpretar la realidad. Así, de acuerdo con Pereira (2011), combinar ambos métodos permitirá desarrollar una mejor comprensión del objeto de estudio. Lo cual fue aplicado al trabajo de campo.

En ese marco, para explorar prácticas, experiencias, opiniones y percepciones, que prevalecen en el mercado empresarial se utilizó una herramienta de investigación 0 evaluación llamada cuestionario. Para mejor asertividad, se utilizó un cuestionario mixto con preguntas cerradas y abiertas (Abarca, Alpízar, Sibaja y Rojas, 2012).

Al respecto, se debió depurar el instrumento estadísticamente, para luego aplicarlo en localidades geográficas de la Región Pacífico Central, a empresarios 0 empresarias de Pymes que desearon participar.

Como una primera etapa, se deseó trabajar con una muestra estadística, pero por la falta de información de las entidades gubernamentales, se decidió realizar un censo empresarial de la región. Al principio del trabajo de campo, se encontró cierta resistencia para obtener la información. No obstante, se logró cubrir un $90 \%$ de la Región Pacífico Central.

Como una segunda etapa, este estudio contó con la participación de estudiantes de TCU, a los cuales se les capacitó e instruyó para la obtención de los datos. Asimismo, se logró visitar a cada empresario (a) en su lugar de trabajo. Como tercera etapa, se realizaron tres depuraciones de datos, para complementar la información final (al principio, a mediados y al final).

La cuarta etapa consistió en realizar las tabulaciones y estadísticas de los resultados que están contenidos en este artículo. Y finalmente, la quinta etapa corresponderá construir una estrategia de acción que contenga talleres socioeducativos, acompañamientos empresariales y asesorías por parte del TCU a futuro con alianzas de entidades públicas, de manera integrada.

En total, se aplicaron 1359 cuestionarios. El objeto de estudio estuvo representado por Pymes formales, legalmente registradas ante las instituciones estatales. Se advierte que las Pymes informales no fueron estudiadas o investigadas en este trabajo.

Esta investigación inició en enero del 2018 y terminó en mayo del 2019. 


\section{RESULTADOS}

Como se indicó anteriormente, se diagnosticaron 1359 Pymes de la Región Pacífico Central en localidades como: Puntarenas Centro, Barranca, Esparza Herradura, Jacó, Quebrada de Ganado, Herradura, Miramar, El Roble, Chacarita y Orotina, las cuales arrojan resultados importantes para meditar sobre la situación de esta población empresarial. Por tanto, presentamos los siguientes resultados, ordenados por las categorías mencionadas al inicio del trabajo:

\subsection{Identificación de la empresa}

Como elemento central, los aspectos generales de una empresa u organización están contenidos en estos apartados, los cuales sirven para identificar lo más relevante de la misma.

\subsubsection{Empresarios de Pymes según género}

A partir de los datos, se presenta el Cuadro I, acerca del género de la población en estudio. Aunque se advierte que es una categoría cualitativa, no deja de ser importante para la investigación.

\section{Cuadro I}

Género de empresarios, 2019

\begin{tabular}{ccc}
\hline & Absoluto & Relativo \\
\hline Hombre & 789 & $58 \%$ \\
Mujer & 570 & $42 \%$ \\
\hline Total & $\mathbf{1 3 5 9}$ & $\mathbf{1 0 0} \%$ \\
\hline
\end{tabular}

Fuente: elaboración propia con base en cuestionarios.

Para efectos de este análisis, un $58 \%$ de los empresarios son hombres, mientras que, un $42 \%$ restante pertenece a mujeres. En cuanto a los datos anteriores, se respalda lo que ocurre internacionalmente, donde el papel de la mujer en los negocios empresariales va en aumento, al igual que en países desarrollados como Estados Unidos y Europa.

\subsubsection{Edad del empresario (a)}

Según el Cuadro II, el rango más sobresaliente es un $26 \%$, el cual está representado por empresarios(as) con edades entre 36 a 45 años. Posteriormente le sigue un rango entre 46 a 55 años con un $25 \%$, un $22 \%$ en edades de 26 a 35 años, un $19 \%$ está constituida por una edad de más de 55 años y, por último, solamente un $8 \%$ poseen una edad de menos de 25 años. 


\section{Cuadro II}

Edad de los empresarios y empresarias, 2019

\begin{tabular}{ccc}
\hline & Absoluto & Relativo \\
\hline Menos de 25 años & 109 & $8 \%$ \\
De 26 a 35 años & 299 & $22 \%$ \\
De 36 a 45 años & 353 & $26 \%$ \\
De 46 a 55 años & 340 & $25 \%$ \\
Más de 55 años & 258 & $19 \%$ \\
\hline Total & $\mathbf{1 3 5 9}$ & $\mathbf{1 0 0 \%}$ \\
\hline
\end{tabular}

Fuente: elaboración propia con base en cuestionarios.

Debe señalarse que en estudios realizados por Global Entrepenurship Monitor en todo el mundo, este perfil demográfico para esta región es muy similar a otros 69 países, pues, personas entre 25 y 34 años presentan una tasa muy alta de actividad emprendedora a esas edades (Landaeta, 2013). En apariencia, las edades de más de 25 años hasta 45 años representan las cifras más altas de este trabajo.

\subsubsection{Nivel de educación}

En cuanto al nivel de educación, el Cuadro III evidencia que un $28 \%$ de los empresarios o empresarias encuestadas poseen educación universitaria completa, es decir, cuentan con un título profesional. En relación a esto, se aclara que muchas veces su especialidad no es el manejo de una empresa. Por otra parte, tanto la secundaria incompleta como la secundaria completa representa un 17\% del total. Asimismo, primaria completa un $13 \%$, el bachillerato universitario completo un $12 \%$, universitaria incompleta un $10 \%$, y por último, primaria incompleta con un $3 \%$.

\section{Cuadro III}

Nivel de educación, 2019

\begin{tabular}{lc}
\hline & Porcentaje \\
\hline Primaria incompleta & $3 \%$ \\
Primaria completa & $13 \%$ \\
Secundaria incompleta & $17 \%$ \\
Secundaria completa & $17 \%$ \\
Bachillerato universitario incompleto & $10 \%$ \\
Bachillerato universitario completo & $12 \%$ \\
Universidad completa & $28 \%$ \\
\hline \multicolumn{1}{c}{ Total } & $\mathbf{1 0 0} \%$ \\
\hline
\end{tabular}

Fuente: elaboración propia con base en cuestionarios. 


\subsubsection{Inicio de operaciones de las Pymes}

En el mundo, ante los grandes cambios de la historia y el progreso de las civilizaciones, el crecimiento económico está relacionado con el desarrollo local de una región. Así, tomando ese escenario como punto de inicio, se dirá que constituir una empresa es una tarea es compleja. Dado que sólo permanecen las que posean las habilidades para lograrlo.

Del Cuadro IV, llama la atención que una significativa mayoría (65\%) se encuentran en un rango entre un 2009 y 2019. Sin embargo, preocupa que el porcentaje mayoritario sean empresas de menos de diez años de estar en el mercado costarricense.

\section{Cuadro IV}

Inicio de las operaciones Pymes- Pacífico Central 2019

\begin{tabular}{ccc} 
Años de creación & Total de empresas por año & Porcentaje \\
$2009-2019$ & 474 & $65 \%$ \\
$1918-2008$ & 885 & $35 \%$ \\
\hline Total & $\mathbf{1 3 5 9}$ & $\mathbf{1 0 0 \%}$ \\
\hline
\end{tabular}

Fuente: Elaboración propia con base en cuestionarios.

Siguiendo el análisis, el restante lo determina un 35\% con empresas que datan desde 1918 hasta el 2008. Aunado a este resultado, en la localidad de Puntarenas, se encuentran empresas de más de cincuenta años de mantenerse en el mercado. Tal es el caso de la Pensión Zagala que lleva más de 100 años al servicio de la sociedad porteña, fundada en 1918.

\subsection{Estructura}

En esta categoría, es primordial destacar que todas las empresas son diferentes y, además, presentan diferentes estructuras en el desempeño de la economía.

\subsubsection{Negocio familiar}

En las tendencias del desarrollo el tipo de empresa es importante, para la generación de empleo en la economía. En el Cuadro $\mathrm{V}$ un $69 \%$ de la población considera que su empresa es familiar porque está administrada por la familia. Mientras que un $31 \%$ indica que su empresa no es familiar, dado que no tienen que ver con las actividades de la Pyme. 


\section{Cuadro V}

Considera que su empresa es familiar, 2019

\begin{tabular}{ccc}
\hline & Absoluto & Relativo \\
\hline Sí & 938 & $69 \%$ \\
No & 421 & $31 \%$ \\
\hline Total & $\mathbf{1 3 5 9}$ & $\mathbf{1 0 0 \%}$ \\
\hline
\end{tabular}

Fuente: elaboración propia con base en cuestionarios.

Estos datos nos indican una realidad nacional que predomina en Costa Rica: Ias empresas familiares. En ese contexto, una de sus desventajas radica en cuanto a la toma de decisiones que muchas veces no son objetivas y podría causar el cierre de la organización (Gutiérrez, Díaz, Romero y Sulbarán, 2017). Por otro, entre sus fortalezas, se encuentra un fuerte vínculo entre la familia y el negocio.

\subsubsection{Tamaño de la Pyme}

Para caracterizar el tamaño de la Pyme, el Cuadro VI suministra que un $87 \%$ de ellas poseen de 1 a 5 colaboradores, un $11 \%$ son empresas pequeñas y un $2 \%$ poseen de 31 a 100 colaboradores dentro de la categorización de la CCSS (2018). Lo anterior corresponde a la realidad costarricense en las cuales el Ministerio de Economía, Industria y Comercio, el cual determina que las empresas de tamaño micro tienden al aumento en la economía (MEIC, 2017).

\section{Cuadro VI}

Cantidad de colaboradores de la Pyme, 2019

\begin{tabular}{|c|c|c|}
\hline & Tipo & Porcentaje \\
\hline De 1 a 5 colaboradores & micro & $87 \%$ \\
\hline De 6 a 30 colaboradores & pequeña & $11 \%$ \\
\hline De 31 hasta 100 colaboradores & mediana & $2 \%$ \\
\hline Total & 1359 & $100 \%$ \\
\hline
\end{tabular}

Fuente: elaboración propia con base en cuestionarios.

Sin embargo, partiendo de esas consideraciones, una pregunta sería ¿Por qué no crecen y pasan de micro $_{8}$ a pequeñas? Entre las respuestas, algunos empresarios o empresarias indicaron que altas cargas sociales y altos impuestos muchas veces las restringe para desarrollarse.

8 Una caracterización de las pymes costarricenses, de acuerdo con las estadísticas de la Caja Costarricense de Seguro Social (CCSS). Sería que para el año 2018, un 74\% de las pymes eran empresas de tipo micro. ¿Qué significa? que una gran mayoría de estas empresas poseen un número limitado de trabajadores o colaboradores, quizás, menos de cinco y disponen de pocos recursos financieros para invertir en tecnología e investigación. Tales condiciones provocan que una mayoría trabaje en niveles de subsistencia y rentabilidad bajas para crecer. 


\subsubsection{Pago oportuno de obligaciones de la Pyme}

Como puede observarse, en el Cuadro VII, las empresas Pymes constituidas, un $95 \%$ de ellas admiten que pagan siempre sus obligaciones o tributos, mientras que, un rango de $3 \%$ las paga casi siempre, y un $1 \%$ las paga a veces y otro $1 \%$ no responde.

\section{Cuadro VII}

Pago oportuno de obligaciones, 2019

\begin{tabular}{lcc}
\hline & Absoluto & Relativo \\
\hline Siempre & 1291 & $95 \%$ \\
Casi siempre & 40 & $3 \%$ \\
A veces & 14 & $1 \%$ \\
No responde & 14 & $1 \%$ \\
\hline \multicolumn{1}{c}{ Total } & $\mathbf{1 3 5 9}$ & $\mathbf{1 0 0 \%}$ \\
\hline
\end{tabular}

Fuente: elaboración propia con base en cuestionarios.

Este Cuadro VII, refleja claramente que, ellas al ser Pymes formales, ${ }_{9}$, pagan de manera oportunamente sus tributos, ya sea con la Municipalidad, Caja Costarricense de Seguro Social (CCSS), Instituto Nacional de Seguros (INS), entre otros. Añadiendo a este punto, varios empresarios argumentaron que el gobierno realiza constantemente visitas a sus empresas por medio del Ministerio de Salud.

\subsection{Actividades desarrolladas}

En esta categoría se analiza la actividad empresarial en la que se encuentra la Pyme, además de la importancia de destacarse en alguna organización o grupo.

\subsubsection{Sector de la economía que se encuentra la Pyme}

Como se puede observar, en el cuadro siguiente, se revela que un $66 \%$ de las Pymes participantes del estudio se dedican al comercio. Mientras que un $29 \%$ a servicios, un $4 \%$ a turismo y un $1 \%$ a la industria. Aunado a esto, en su mayoría son empresas de tipo micro, como se reveló anteriormente. Examinemos el siguiente Cuadro VIII:

9 En el país, en el año 2002, creó la Ley 8262 Fortalecimiento de las pequeñas y medianas empresas, la cual establece que las pymes deben cumplir con las cargas sociales, tributarias y obligaciones laborales. Además, que existen instituciones públicas que las promueven como el Ministerio de Economía, Industria y Comercio. Aunado a esto, en la tesis de doctorado de Cousin (2015), se encuentra un estudio más profundo de la ley en el marco jurídico y sus condiciones. 


\section{Cuadro VIII}

Empresas por rama de actividad, 2019

\begin{tabular}{|c|c|c|}
\hline & Absoluto & Relativo \\
\hline Comercio & 896 & $66 \%$ \\
\hline Servicios & 394 & $29 \%$ \\
\hline Turismo & 69 & $4 \%$ \\
\hline Total & 1359 & $100 \%$ \\
\hline
\end{tabular}

Fuente: elaboración propia con base en cuestionarios.

En ese orden de ideas, precisamente, el Ministerio de Economía Industria y Comercio (MEIC, 2017), declara que mayoritariamente, Costa Rica es un país dedicado al comercio.

Como un elemento más, diversos estudios argumentan que la actividad de comercio y servicios es la actividad empresarial en donde participan la mayoría de las Pymes costarricenses (Navarro, 2015). Sin embargo, se deben tomar acciones para no descuidar y fortalecer estas actividades comerciales, dado que las empresas grandes con dinamismos comerciales más especializados, representan una competencia muy alta.

\subsubsection{Participación en alguna organización o grupo}

Básicamente, las Pymes encuestadas, según el Cuadro IX, un $91 \%$ indican que la mayoría no pertenecen a ninguna organización 0 grupo, luego un 4\% asegura formar parte de una asociación y otro 4\% pertenece a una cámara. Finalmente, solo un $1 \%$ pertenece a una fundación. Entre sus razones, el $91 \%$ manifiesta que, generalmente, se encuentran solas en el mercado, tratando de hacer lo que pueden por sobrevivir; además, no se promocionan estas organizaciones entre ellas.

\section{Cuadro IX}

Pertenencia a una organización o grupo, 2019

\begin{tabular}{ccc}
\hline & Absoluto & Relativo \\
\hline Ninguna & 1236 & $91 \%$ \\
Asociación & 54 & $4 \%$ \\
Cámara & 54 & $4 \%$ \\
Fundación & 15 & $1 \%$ \\
\hline Total & $\mathbf{1 3 5 9}$ & $\mathbf{1 0 0 \%}$ \\
\hline
\end{tabular}

Fuente: elaboración propia con base en cuestionarios.

Sería recomendable que el gobierno estimulara estas organizaciones en sus políticas públicas, para crear mayores encadenamientos productivos en la Región del Pacífico Central, porque es preocupante una mayoría significativa tan alta (91\%) en la negativa de este proceso; quizás por desconocimiento. 


\subsection{Administración de la Información}

Desde otra perspectiva, es importante analizar los indicadores de la información en la cual se desenvuelve la empresa para evaluar sus respaldos y la existencia de mantenimiento 0 soporte de la información en la toma de decisiones.

\subsubsection{Sistema contable}

En esa línea de análisis, este estudio revela que un $64 \%$ de los empresarios 0 empresarias son los encargados de tomar las decisiones, al mismo tiempo controlar el inventario y otros temas gerenciales. En ese marco, un 35\% de los participantes afirman que el encargado de realizar estas actividades es el contador y un $1 \%$ indicó no estar de acuerdo revelar esta información a terceros. A grandes rasgos, preocupa para la investigación que un 35\% de la población no tome sus decisiones empresariales para la buena marcha de su negocio y eso pueda ocasionar momentos de fracasos en algún momento de su gestión.

\subsection{Información del Mercado}

Ciertamente, el Mercadeo engloba acciones, estrategias, elementos y tácticas empresariales que estimulan las ventas y objetivos de toda empresa, aunado a un buen equipo de trabajo.

\subsubsection{Conocimiento del mercado}

De acuerdo con el Cuadro X, la mayoría de los empresarios encuestados explican que poseen bastante conocimiento del mercado donde se encuentran, tratándose así de un $76 \%$, mientras que un $20 \%$ poseen un conocimiento regular, un $3 \%$ muy poco, y un $1 \%$ añade que no posee ningún conocimiento. Examinemos el Cuadro $X$ a continuación:

\section{Cuadro X}

Conocimiento del Mercado, 2019

\begin{tabular}{|c|c|c|}
\hline & Absoluto & Relativo \\
\hline Bastante & 1033 & $76 \%$ \\
\hline Regular & 279 & $20 \%$ \\
\hline Muy poco & 41 & $3 \%$ \\
\hline Nada & 6 & $1 \%$ \\
\hline Total & 1359 & $100 \%$ \\
\hline
\end{tabular}

Fuente: elaboración propia con base en cuestionarios.

Inquieta en este estudio, el porcentaje de la población con respuestas como: regular $20 \%$, muy poco $3 \%$ y nada $1 \%$, por las demandas de la economía y los cambios abruptos que se presentan en el mercado empresarial. 


\subsubsection{Conocimiento del grado de competencia}

Ahora bien, en el mundo, diversos factores mueven la globalización y las organizaciones deben de responder competitivamente a los cambios del entorno. En nuestros tiempos, los clientes son más exigentes que hace 30 años: muchos desean hacer pedidos o preguntas por redes sociales u otro medio informativo sobre la empresa. Todos estos cambios hacen que se transformen las maneras de vender y la fuerza de ventas en cada empresa deban ser más agresivas. A esto, Costa Rica no es la excepción y, por tal motivo, se presenta el siguiente cuadro:

\section{Cuadro XI}

Percepción sobre el grado de competencia, 2019

\begin{tabular}{lcc}
\hline & Absoluto & Relativo \\
\hline Alta & 721 & $53 \%$ \\
Media & 490 & $36 \%$ \\
Baja & 108 & $8 \%$ \\
No existe & 40 & $3 \%$ \\
\hline \multicolumn{1}{c}{ Total } & $\mathbf{1 3 5 9}$ & $\mathbf{1 0 0 \%}$ \\
\hline
\end{tabular}

Fuente: elaboración propia con base en cuestionarios.

En el Cuadro XI, puede analizarse que, para la percepción del grado de competencia, por una parte, un $53 \%$ afirman que consideran la competencia alta, por otra parte, un $36 \%$ de la población empresarial considera que es media, mientras que un $8 \%$ considera que es baja y un $1 \%$ que no existe. De acuerdo con estos datos, es importante señalar que urgen medidas importantes en los acompañamientos empresariales para enfrentar la competencia y otros retos. Aunque, más de la mitad (53\%) está consciente de la competencia, éste porcentaje debería ser mayor para este contexto. Dado que, se ocupan diferentes estrategias para contrarrestarla.

\subsubsection{Conocimiento de los clientes}

Prosiguiendo la investigación, al consultar sobre el conocimiento de sus clientes, el Cuadro XII sintetiza que un $77 \%$ afirma conocer "siempre" hacia cuales clientes van dirigidos sus productos. Por otra parte, un $19 \%$ afirmaron casi siempre conocerlos y un $4 \%$ a veces. Veamos el Cuadro XII:

\section{Cuadro XII}

Conocimiento sobre los clientes, 2019

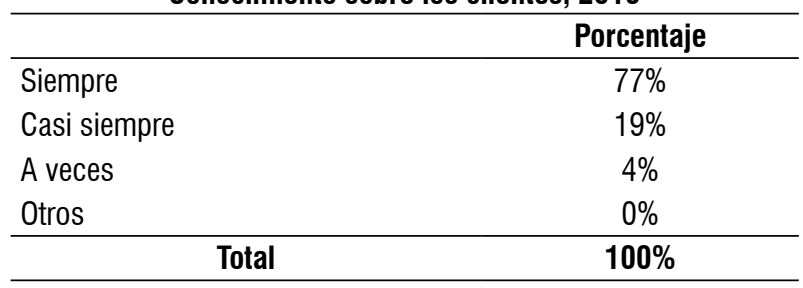

Fuente: elaboración propia con base en cuestionarios. 
Este apartado es importante, dado que, según sus respuestas, conocen al cliente, así como sus gustos y preferencias. Sin embargo, no basta conocer los clientes, hoy se deben atraer hacia los objetivos de la organización para "encantarlos" estableciendo una relación de largo plazo.

\subsubsection{Características que diferencian sus productos}

Al estudiar los productos o servicios que ofrecen estas empresas, se presentan los siguientes resultados. Primero, un 35\% se distingue de la competencia por su calidad, segundo un $29 \%$ por el precio, tercero un $24 \%$ por el servicio y por último un $12 \%$ menciona diferenciarse por su exclusividad. Sin embargo, admiten, en su mayoría, que la falta de recursos financieros las limita, dado que no son en su mayoría sujetos de crédito ${ }_{10}$ en los bancos y eso les impide crecer para ampliar el negocio. Examinemos el Cuadro XIII:

\section{Cuadro XIII}

Características de sus productos, 2019

\begin{tabular}{lc}
\hline & Porcentaje \\
\hline Calidad & $35 \%$ \\
Precios & $29 \%$ \\
Exclusividad & $12 \%$ \\
Servicio & $24 \%$ \\
\hline \multicolumn{2}{c}{ Total } \\
\hline
\end{tabular}

Fuente: elaboración propia con base en cuestionarios.

\subsubsection{Publicidad}

Las grandes empresas empezaron, en el mundo empresarial como micro empresas. Posteriormente, muchas que lograron sus éxitos lo hicieron en base a un eficiente plan de Mercadeo. En ese sentido, existen diversas clases de promocionar un servicio o producto, al igual que promocionar la organización. Veamos el siguiente cuadro:

10 Para una aclaración en materia de créditos los bancos estatales costarricenses están obligados a fomentar el sector de las pymes. Desde ese marco, para el 2008, se creó la Ley 8634 Sistema de Banca para el desarrollo (SBD), en la cual el Estado principia que las pymes posean una herramienta crediticia, pero únicamente les financia un $75 \%$ de sus bienes. Es decir, sin esos recursos no son sujetos de crédito; porque no cuentan con subsidios del gobierno como en otros países latinoamericanos como Chile. 


\section{Cuadro XIV}

Forma de promocionar sus productos, 2019

\begin{tabular}{lc}
\hline & Porcentaje \\
\hline Publicidad de boca en boca & $47 \%$ \\
Publicidad en redes sociales & $26 \%$ \\
Promociones de ventas & $5 \%$ \\
Todas las anteriores & $22 \%$ \\
\hline \multicolumn{1}{c}{ Total } & $\mathbf{1 0 0 \%}$ \\
\hline
\end{tabular}

Fuente: elaboración propia con base en cuestionarios.

Se observa en el Cuadro XIV que la forma más utilizada por las Pymes para promocionarse es mediante la publicidad de boca en boca con un 47\%, seguidamente por la publicidad en redes sociales con un $26 \%$, y solo el $5 \%$ dice promocionarse por medio de promociones en ventas, el otro $22 \%$ afirma utilizar todas las opciones anteriores.

Según el análisis de los datos obtenidos, al consultar ¿Por qué no utilizaban la publicidad en redes sociales? Su respuesta fue que algunos desconocían sus usos y ventajas. Sin embargo, es importante que este tipo de publicidad se implemente en ellas dado que permite que su promoción sea más efectiva y esto, generaría una cantidad de personas o clientes al tanto de sus bienes o servicios y, además, se obtendría sin hacer inversión muy costosa.

\subsubsection{Valor de las ventas netas 0 ingresos netos}

Si bien es cierto, las empresas están insertas en un tejido empresarial, se requiere que sus gestiones gerenciales produzcan un bien o servicio con un rendimiento financiero en las operaciones. Tal y como se observa en el Cuadro XV, en relación con las ventas netas anuales, un $40 \%$ de la población estudiada indica que posee ventas netas anuales menores a 20 mil dólares $_{11}$, 0 sea, menos de 11 millones de colones en Costa Rica. A la vez, un 35\% de empresarios que prefieren no responder esta información y omitirla, seguida de un $13 \%$ que admite que sus ingresos oscilan entre $\$ 21$ mil y 50 mil dólares. Seguido por un 8\% restante a empresas que tiene ventas netas superiores a los $\$ 86.000$ dólares y para terminar un $4 \%$ admite que sus ventas netas oscilan entre $\$ 51$ mil a 85.000 dólares en adelante.

11 Tipo de cambio del Banco Central de Costa Rica al 31 de mayo del 2019, un dólar $=590.54$ en colones costarricenses. (Banco Central, 2019). 


\section{Cuadro XV}

Valor de las ventas netas anuales, 2019

\begin{tabular}{lll}
\hline Descripción & Absoluto & Relativo \\
\hline Menos de \$ 20.000 dólares & 550 & $40 \%$ \\
Más de \$21 hasta 50.000 dólares & 178 & $13 \%$ \\
Más de \$51 hasta 85.000 dólares & 46 & $4 \%$ \\
De 86.000 dólares en adelante & 115 & $8 \%$ \\
No responde & 470 & $35 \%$ \\
\hline Total & 1359 & 100 \\
\hline
\end{tabular}

Fuente: Elaboración propia con base en cuestionarios.

En lo económico, se analiza que el porcentaje mayor está representado por ventas netas menores a 11 millones de colones, casi en un nivel de subsistencia y, por ende, esto sería bajo crecimiento en ellas, así como limitaciones importantes para invertir en tecnología y desarrollo.

Por otro lado, las Pymes que no respondieron (35\%) podrían aumentar ese porcentaje del 40\%. Lo anterior, resulta preocupante para la economía de la Región del Pacífico Central que ocupa crecer económicamente.

\subsection{Mejora continua}

De acuerdo con la percepción de los empresarios o empresarias de las Pymes. Existen áreas que se deben de mejorar o reforzar para su buena gestión.

\subsection{1 Áreas de la empresa que se desea mejorar}

A grandes rasgos, el Cuadro XVI evidencia que las áreas que la población desea mejorar, en orden de importancia, se encuentra: Mercadeo con un 32\%, seguida por un porcentaje de $29 \%$ en la parte Financiera $29 \%$, la Administrativa con un $25 \%$. Para terminar, un $12 \%$ asegura que necesitan mejorar su área Gerencial y un $2 \%$ indica que serían otras áreas.

\section{Cuadro XVI}

Áreas de la empresa por mejorar, 2019

\begin{tabular}{lc}
\hline \multicolumn{1}{c}{ Tipo } & Porcentaje \\
\hline Mercadeo & $32 \%$ \\
Financieras & $29 \%$ \\
Administrativas & $25 \%$ \\
Gerenciales & $12 \%$ \\
Otra & $2 \%$ \\
\hline \multicolumn{2}{c}{ Total } \\
\hline
\end{tabular}

Fuente: elaboración propia con base en cuestionarios. 
De la información anterior, se puede analizar que, las empresas deben considerarse desde la eficiencia y eficacia. Asimismo, queda claro que no están aisladas, dado que dependen del entorno para poder producir sus bienes o servicios. Sin embargo, para este sector costarricense es necesario invertir en un mejoramiento continuo para lograr las metas propuestas y buscar nuevas alianzas para que otras instituciones, como el Ministerio de Economía e Industria (MEIC), el Instituto Nacional de Aprendizaje (INA), la Universidades públicas como: Tecnológico de Costa Rica (TEC), Universidad Nacional, Universidad Nacional (UNA), Universidad Técnica Nacional (UTN), entre otras, nos acompañen a realizar tareas más efectivas. Dado que Costa Rica no cuenta con un departamento designado formalmente para que las acompañe empresarialmente en su gestión, como lo posee Chile con CORFO (Corporación de Fomento para la Producción) y SERCOTEC (Servicio de Cooperación Técnica), entre otros.

\subsubsection{La empresa ha recibido algún tipo de capacitación}

En respuesta a esta pregunta, de acuerdo con el Cuadro XVII, más de la mitad de las empresas encuestadas, 0 sea, un 74\%, afirman nunca haber recibido alguna capacitación para mejorar la administración de su empresa, mientras que, el $26 \%$ afirmó sí haberla recibido, pero de manera privada. 0 sea, no fueron instituciones públicas quienes se las suministraron.

\begin{tabular}{ccc}
\multicolumn{2}{c}{ Cuadro XVII } \\
& La empresa ha recibido alguna capacitación, 2019 \\
\hline & Respuesta & Porcentaje \\
\hline Sí & & $26 \%$ \\
No & $74 \%$ \\
\hline Total & $\mathbf{1 0 0 \%}$ \\
\hline
\end{tabular}

Fuente: elaboración propia con base en cuestionarios.

En este caso, se evidencia la necesidad de que ciertas instituciones promuevan asesorías y acompañamientos integrados, ya que, esto podría mejorar las utilidades de las empresas, mejorando así la economía de la región en estudio y fortalecer su capital humano.

Asimismo, una mayoría significativa insiste en la importancia de estas acciones, ya que muchos de los empresarios fundaron sus empresas con conocimientos muy básicos en áreas como administración, finanzas, mercado y gerencia.

\subsubsection{Necesidades específicas de las Pymes}

En cuanto a estas necesidades de acompañamiento y asesorías, en las áreas específicas, de acuerdo con el Cuadro XVIII, se observa lo siguiente: 


\section{Cuadro XVIII}

Necesidades específicas, 2019

\begin{tabular}{lc}
\hline & Porcentaje \\
\hline Atención al Cliente & $19 \%$ \\
Elaboración de Página WEB & $15 \%$ \\
Contabilidad & $15 \%$ \\
Manejo de inventarios & $14 \%$ \\
Plan de Negocios & $14 \%$ \\
Ninguno & $10 \%$ \\
Todos los cursos & $9 \%$ \\
Recursos Humanos & $2 \%$ \\
Otros & $2 \%$ \\
\hline Total & $\mathbf{1 0 0 \%}$ \\
\hline
\end{tabular}

Fuente: elaboración propia con base en cuestionarios.

De todo lo anterior, el Cuadro XIV expone que la principal necesidad específica se encuentra en "Atención al cliente", con un 19\%, la cual es la más solicitada. Posteriormente, la "Elaboración de página web" y "Contabilidad" representan un $15 \%$ cada una, y en tanto "Manejo de inventarios" como "Plan de negocios" con 14\%. Asimismo, un 9\% de las empresas estarían dispuesto a recibir todas las capacitaciones, anteriormente mencionadas, mientras que, las menos requerida, fue Presupuesto y Recursos Humanos ambas con $2 \%$.

\section{CONCLUSIONES}

De todo lo anterior, esta investigación evidencia una serie de variables e indicadores de las Pymes de la Región Pacífico Central, de Costa Rica, en las cuales el Trabajo Comunal Universitario-653, intentará tomar acciones para mitigar sus debilidades y amenazas, y a futuro potenciar las fortalezas.

En este estudio se desprenden las siguientes conclusiones para la Región Pacífico Central:

- De la investigación, en cuanto al género del empresario o empresaria, la mayoría de las micro, pequeñas y medianas empresas de la Región del Pacífico Central, presentan que un $58 \%$ pertenecen a hombres y $42 \%$ son mujeres.

- De igual forma, en relación con la edad, en la población estudiada revela que su promedio oscila entre 36 a 45 años.

- $\quad$ En cuanto al nivel de educación, un $28 \%$ de los participantes del proyecto poseen educación universitaria completa, pero, con la limitación que sus estudios no necesariamente son en Administración. Por otro lado, 
tanto secundaria incompleta, como la secundaria completa, representan un $17 \%$ cada una del total. En esa línea, la primaria completa arroja un resultado de un $13 \%$, asimismo el bachillerato universitario completo $12 \%$, universitaria incompleta un 10\%. De igual forma, en este análisis, la primaria incompleta es muy baja un 3\%. A grandes rasgos, se indica que la mayoría poseen estudios avanzados o profesionales.

- En general, en cuanto al inicio de las operaciones de las Pymes, un 65\% se encuentra entre los años 2009 y 2019. Analizando esta situación, puede indicarse que una empresa consolidada es la que posee más de diez años en el mercado.

- Además, un 35\% se ubica en empresas creadas desde 1918, hasta el 2008. Para este caso la Región Pacífico Central presenta un 35\% que poseen más de este tiempo. Entre sus logros estas Pymes han tenido que competir fuertemente con las tendencias del entorno y la tecnología.

- Más aún, es significativo acentuar que, en el distrito de Puntarenas, se encuentran empresas de más de cincuenta años de permanecer en el mercado, tal es el caso de la Pensión la Zagala que lleva más de 100 años al servicio de la sociedad porteña, dado que, fue fundada en 1918.

- Un $69 \%$ de la población empresarial reflexiona que la empresa es un negocio familiar porque es administrada por la familia.

- En relación con la actividad económica, el estudio demuestra que un $66 \%$ de ellas pertenece al sector comercio, la parte de servicios $29 \%$, un $4 \%$ a turismo y un 1\% a la industria. En esa línea, el sector comercio es la actividad empresarial que más practican las empresas de esta Región Pacífico Central, al igual que el resto del país. Aunque el TCU-653 podría evaluar acciones para que la actividad de turismo pueda incrementarse, en relación con la belleza escénica de las zonas y dar un significado de actividades más diferente en esas localidades.

- En cuanto al tamaño de la empresa, se examinó que las microempresas representan la mayor parte de empresas en la región del Pacífico Central representan un $87 \%$, seguido por un $11 \%$ de empresas pequeñas y $2 \%$ empresas medianas. Esta situación es muy similar a la situación general en Costa Rica, en donde las micro empresas son las más numerosas.

- En cuanto al pago oportuno de sus obligaciones un 95\% considera estar al día.

- Ahora bien, un $91 \%$ de las Pymes admiten no pertenecer a alguna organización o grupo. Entre sus razones manifiestan que desconocen este tipo de grupos, pues poseen poca información sobre los mismos. En los países ricos la asociatividad empresarial, es muy común para 
poder ejercer una articulación y realizar venta de productos o servicios a empresas grandes o inclusive al gobierno. Sería recomendable que el Estado fomentara esta iniciativa, para en un futuro, estas Pymes sean articuladas y se empoderen los encadenamientos productivos de la región.

- Básicamente, este estudio revela con relación que, en cuanto a la administración para la toma de decisiones. Generalmente, un 64\%; menciona que es el dueño es el encargado financiero de tomarlas, además de controlar el inventario y otros temas empresariales. Asimismo, un $35 \%$ de los participantes afirman que el encargado de realizar estas actividades es el contador y un 1\% indicó no estar de acuerdo reflejar esta información a terceros. Sin embargo, sería recomendable que en un futuro todos los propietarios o propietarias de las Pymes tomen sus decisiones empresariales, dado que, un $35 \%$ no lo hace.

- Básicamente, un 76\%, de la población considera que poseen un considerable conocimiento del mercado donde se encuentran. Mientras tanto, un $20 \%$ indican tener un conocimiento regular, un 3\% muy poco, y un $1 \%$ añade que no posee ninguno. Preocupa en este estudio, como se indicó antes que el porcentaje de empresarios con percepciones como: regular $20 \%$, muy poco $3 \%$ y nada $1 \%$, tengan este tipo de respuesta, por las vulnerabilidades de la economía y la agresividad del entorno donde se encuentran. Aquí es donde el proyecto de TCU-653, puede llegar a lograr cambios significativos en las expectativas de crecimiento de estas Pymes.

- En relación con la percepción de la competencia entre las Pymes un 53\% expresa que es alta y el restante se divide en un $36 \%$ opina que es media, un $8 \%$ la considera y un $3 \%$ no existe. Al igual que el apartado anterior, es recomendable estar al tanto de la competencia, los cambios que hacen y las tecnologías que implementan, para enfrentar adecuadamente.

- Un $77 \%$ de la población afirma conocer siempre hacia cuáles clientes van dirigidos sus productos, por otra parte, un 19\% asegura que "casi siempre conocerlos", y un $4 \%$ a veces.

- Ahora bien, los productos o servicios que ofrecen estas empresas, se distinguen según el estudio en calidad 35\%, seguida por el precio con $29 \%, 24 \%$ servicio y un $12 \%$ dice diferenciarse por su exclusividad. Esto representa una fortaleza en cuanto a la competencia, dado que el factor calidad es muy importante en la gestión empresarial de estas Pymes.

- $\quad$ En cuanto a la forma de promocionarse, la forma más utilizada por las Pymes es la publicidad de boca en boca con una 47\%, seguidamente, por la publicidad en redes sociales con un $26 \%$, y solo el $5 \%$ dicen promocionarse por medio de promociones en ventas, el otro $22 \%$ afirma 
utilizar todas las opciones anteriores. De lo anterior, preocupa que no se realice una publicidad más formal, para atraer clientes potenciales. Sin embargo, con base en esta debilidad el TCU 653, realizará una estrategia de acción.

- $\quad$ Sobre el apartado de las ventas netas anuales o ingresos netos, un $40 \%$ de la población empresarial revela que posee ventas netas anuales menores a 20 mil dólares. A la vez, un 35\% de empresarios que prefieren no responder esta información y omitirla. Seguida de un $13 \%$ que admite que sus ingresos oscilan entre $\$ 21$ mil y 50 mil dólares. Seguido por un $8 \%$, que posee ventas netas superiores a los $\$ 86.000$ dólares y para terminar un $4 \%$ admite que sus ventas netas oscilan entre $\$ 51$ mil a 85.000 dólares en adelante. Como se analizó en los resultados con ingresos muy bajos, muchas de las Pymes se encuentran en un nivel de subsistencia, por su baja rentabilidad. Además, algunas señalan que ese resultado (40\%), se origina por las pocas oportunidades que se les presentan en el país. Lo anterior, resulta preocupante para la economía de la Región del Pacífico Central que ocupa crecer económicamente.

- En relación con las áreas de la empresa que se desea mejorar, un 32\% admite que deben ser en la parte de Mercadeo, un 29\% expresa que son Financieras, un 25\% manifiesta que más bien, debe ser la Administrativa y otras expresan que son Gerenciales $12 \%$ y, por último, un 2\% señalan otras áreas. El Trabajo Comunal Universitario, intentará definir exactamente las expectativas de estas empresas para poder dar asesorías y acompañamientos efectivos para llegar quizás a un mejoramiento continuo, dado que, como se mencionó el país no cuenta con una entidad formalmente, que las asesore y acompañe específicamente en sus gestiones como lo hace Chile.

- Aunque hay ciertas políticas públicas costarricenses que benefician las Pymes, se necesitan más acciones concretas para fomentarlas y desarrollarlas en sus procesos productivos dado que, sólo un $26 \%$ ha recibido capacitaciones empresariales por parte del gobierno.

- Del estudio se desprende que, en la Región Pacífico Central, un 82\% de la población empresarial está dispuesta a recibir asesorías y acompañamientos empresariales.

- $\quad$ En resumen, la principal necesidad de asesorías y acompañamientos a las Pymes, se determina sobre temas como: "Atención al cliente" con un $19 \%$ siendo la más solicitada según la investigación. Posteriormente, la "Elaboración de página web" y "Contabilidad" representan un 15\% cada una, y en tanto "Manejo de inventarios" como "Plan de negocios" con 14\%. Asimismo, un 9\% de las empresas estarían dispuesto a recibir todas los acompañamientos y asesorías anteriormente mencionadas, mientras que, las menos requerida fue Presupuesto y Recursos Humanos ambas con $2 \%$. 
En relación a esta investigación, el gobierno costarricense debe crear políticas públicas adecuadas que estimulen en las Pymes mejores oportunidades en cuanto al emprendimiento, innovación y competitividad, con el objetivo de promover mejores oportunidades para la sociedad que busca ser en algún momento un país desarrollado. Aunado a esto, se debe promover una cultura empresarial de cambio continuo en mejora y tecnológica porque ellas enfrentan una alta competencia tanto interna como externa. Lo anterior se respalda, porque muchas de ellas, operan con baja rentabilidad, que no les permite elevar a otros niveles sus actividades y proyectos. Por esa razón, muchas trabajan bajo un mercadeo básico y rudimentario con ingresos anuales menores a los 20 mil dólares, 0 sea, 11 millones de colones costarricenses. Como se presentó en el estudio.

Para terminar, el proyecto de Trabajo Comunal Universitario (TCU-653), posee como reto para los años 2020-2021, coordinar y articular mejores esfuerzos interinstitucionales para una mejora empresarial de las Pymes del Pacífico Central en Costa Rica. Los resultados de estas acciones serian presentadas en una investigación posterior.

\section{BIBLIOGRAFÍA}

ABARCA, A., Alpízar F., Sibaja, G. y Rojas, C. (2012). Técnicas cualitativas de investigación. San José: editorial Universidad de Costa Rica.

ASAMBLEA Legislativa: (2002). Ley 8.262 de fortalecimiento de las pequeñas y medianas empresas. San José: Imprenta Nacional. (2008). Ley 8.634 Sistema de Banca para el desarrollo. San José: Imprenta Nacional.

BANCO Central de Costa Rica (2019). Versión electrónica https://www.bccr. fi.cr/SitePages/default.aspx. Consultado 23 de octubre del 2019.

CAJA Costarricense de Seguro Social (2018). Versión electrónica: https:// www.ccss.sa.cr/main_estadisticas Consultado: 20-03-2019.

CARVAJAL, A. (2014). Las convergencias entre Ciencia, Tecnología y Desarrollo. San José: Editorial El Guayacán.

CHEN, S. y García K. (2012). Diagnóstico de las micro, pequeñas y medianas empresas en distritos de Orotina, Montes de oro, El Roble, Barranca, Chacarita, Puntarenas, Esparza y Jacó. Revista de Ciencias Económicas, 30, p.

COUSIN, K. (2015). Estudio de la evolución histórica- económica de las Pymes en Costa Rica (1950-2013). Tesis de Doctorado de Estudios de la Sociedad y la Cultura. San José: Universidad de Costa Rica. 
COUSIN, K. (2018). La pequeña propiedad y mediana propiedad y sus antecedentes a las Pymes de Costa Rica (1813-1920). Disponible en: https://revistacienciassociales.ucr.ac.cr/images/revistas/RCS162/09COUSIN.pdf Consultado 29-08-2019.

INEC. (2015). Indicadores democráticos cantonales 2013. Disponible en: www.inec.go.cr/wwwisis/documentos/INEC/Indicadores_Demograficos_ Cantonales/Indicadores_Demograficos_Cantonales_2013.pdf. Consultado 28-10- 2019.

INEC. (2018). Costa Rica en cifras. Consultado el 28 de febrero 2019 en http://www.inec.go.cr/sites/default/files/documetos-biblioteca-virtual/ recostaricaencifras2018.pdf

GUTIÉRREZ J., Romero J., Díaz M. y Sulbarán N. (2017). Emprendimiento como fuente de desarrollo de la empresa familiar. Algunas reflexiones sobre Venezuela. Revista de Ciencias Sociales. Volumen XXIII, No. 4, Octubre-Diciembre, pp. 98-107.

LANDAETA, M. (2013). El perfil del emprendedor en Costa Rica. San José: Periódico el Financiero. Disponible en: https://www. elfinancierocr.com/Pymes/el-perfil-del-emprendedor-de-costa-rica/ L2F2UNU3IZEOBAOFVNRVSJJ7PQ/story/ Consultado el 20-03-2019.

MEIC. (2017). Aumenta el número de empresas PYMES que le venden al Estado. Consultado el 23 de febrero 2019 en https://www.meic.go.cr/ meic/comunicado/847/aumenta-numero-de-empresas-Pymes-que-levenden-al-estado.php

MEIC. (2017). Estado de Situación de las Pymes en Costa Rica 2016. Consultado el 01 de marzo 2019 en http://reventazon.meic.go.cr/ informacion/Pyme/2017/informe.pdf

MIDEPLAN. (2017). Costa Rica Índice de Desarrollo Social 2017. Disponible en: http://www.conicit.go.cr/biblioteca/publicaciones/publica_cyt/informes/ Indice_Desarrollo_Social_2017.pdf Consultado el 28 -07- 2019.

MIDEPLAN. (2019). Plan Nacional de Desarrollo e Inversión Pública. Consultado el 01 de marzo en https://sites.google.com/expedientesmideplan.go.cr/ pndip-2019-2022/

NAVARRO, L (2015). Comercio y Servicios concentran el 84\% de las Pymes en Costa Rica. Disponible en: https://www.nacion.com/economia/negocios/ comercio-y-servicios-concentran-el-84-de-las-Pymes-de-costa-rica/ HXNMZ6RSURAITEMSO2WAURLJSI/story/ Accesado 25-03-2019. 
OBSERVATORIO de MiPymes: (2010). Hacia el estado de las MiPymes: Primer diagnósticoNacionaldeMiPymes.CostaRica:UniversidadEstatalaDistancia. (2010).Políticas Públicas y MiPyme: ¿Entramado de problemas 0 compartimientos estancos? San José: Universidad Estatal a Distancia. (2010). Informe técnico: Primer Diagnóstico Nacional de MiPymes. San José: Universidad Estatal a Distancia.

PEREIRA, Z. (2011). Los diseños de método mixto en la investigación en educación: Una experiencia concreta. Revista Electrónica Educare Vol. XV (1), 15-29. Consultado el 20-01-2020. Disponible en: https://www. redalyc.org/articulo.oa?id $=194118804003$

SAMPIERI, R., Méndez, S., Mendoza C. y Cuevas Ana (2017). Fundamentos de investigación. Distrito Federal: Editorial Mc Graw Hill.

UNIVERSIDAD de Costa Rica (2018). Reglamento de trabajo comunal universitario Consultado el 20-01-2019. Disponible en: http://www. cu.ucr.ac.cr/normativ/trabajo_comunal.pdf 